Case Report

\title{
Left Atrial Mass Invasion from Pulmonary Neoplasm Extension via the Right Upper Pulmonary Vein Presenting as Ipsilateral Stroke
}

\author{
Piercarlo Ballo, ${ }^{1}$ Raffaele Laureano, ${ }^{2}$ Mariapia Briganti, ${ }^{2}$ Maria Teresa Passaleva, ${ }^{2}$ \\ Fiorella Piani, ${ }^{2}$ Cecilia Piga, $^{2}$ Stefano Tatini, ${ }^{2}$ and Giovanni Maria Santoro ${ }^{1}$ \\ ${ }^{1}$ Cardiology Unit, S. Maria Annunziata Hospital, Florence, Italy \\ ${ }^{2}$ Department of Medicine, S. Maria Annunziata Hospital, Florence, Italy
}

Correspondence should be addressed to Piercarlo Ballo; pcballo@tin.it

Received 31 July 2016; Revised 15 November 2016; Accepted 20 November 2016

Academic Editor: Jagdish Butany

Copyright (C) 2016 Piercarlo Ballo et al. This is an open access article distributed under the Creative Commons Attribution License, which permits unrestricted use, distribution, and reproduction in any medium, provided the original work is properly cited.

Left atrial invasion by lung cancer via haematogenous pathways is a relatively uncommon but potentially life-threatening event. While several cardiac complications of cardiac involvement have been previously described, the evolution towards cerebral stroke has been rarely reported. In this case report, we describe an atypical case of haematogenous metastatic invasion of the left atrium from pulmonary neoplasm extension presenting as an ipsilateral stroke whose ASCO classification changed during the clinical management.

\section{Introduction}

Although cardiac metastases have been reported in up to $25 \%$ of patients with lung cancer in autoptic studies [1], the detection of cardiac involvement in these patients is relatively uncommon in clinical practice [2-4]. The metastatic pathway to the heart is often lymphatic, but hematogenous patterns can also be observed [5]. From a clinical point of view, invasion of the left heart may be a life-threatening event, potentially leading to a number of complications such as obstructed pulmonary venous flow [6], cardiac tamponade [7], ventricular arrhythmias [8], complete atrioventricular block [9], left ventricular inflow obstruction [10], and myocardial infarction [11].

Cerebral stroke as a result of systemic embolization from the left heart has been exceptionally reported [12] and may sometimes represent the first clinical presentation of the neoplasm. Adequate identification of the underlying cause of stroke is therefore of major clinical importance in these cases. Compared with the old Trial of ORG 10172 in Acute Stroke Treatment (TOAST) classification system, newer classification schemes such as the ASCO (A, atherosclerosis; $\mathrm{S}$, small vessel disease; $\mathrm{C}$, cardiac source; $\mathrm{O}$, other causes) phenotypic system can facilitate the identification of the most likely cause by grading the probability of each factor and accounting for the extent of the diagnostic work-up [13]. This approach may be particularly useful when multiple potential mechanisms are present, since it reduces the prevalence of patients with stroke of indeterminate origin. In this report, we describe an unusual case of left atrial (LA) invasion from pulmonary neoplasm extension via the right upper pulmonary vein whose first clinical presentation was characterized by an ipsilateral stroke with evolving ASCO categorization during the management.

\section{Case Report}

A 76-year-old man presented to the Emergency Department because of left hemiparesis and dysarthria. His history was relevant for past smoking, systemic hypertension, and laryngeal cancer treated by total laryngectomy and tracheostomy 5 years earlier. His usual therapy included aspirin, losartan, and doxazosin. During the last 3 weeks, he had shown recurrent episodes of postural instability and paresthesia in the left arm. A brain computed tomography (CT) performed 2 weeks earlier had shown no significant abnormalities. At 


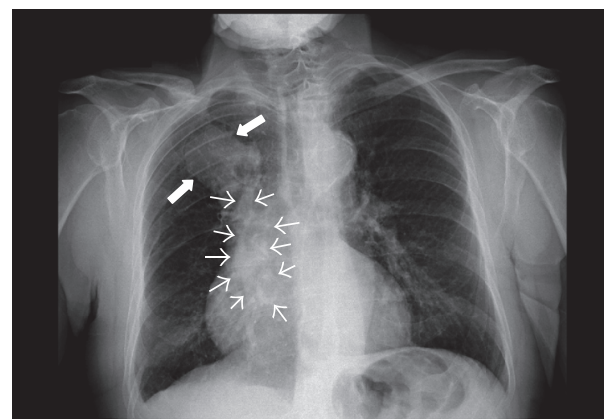

FIGURE 1: Chest X-ray showing a rounding mass projecting over the right upper lobe and extending towards the right upper portion of cardiac silhouette.

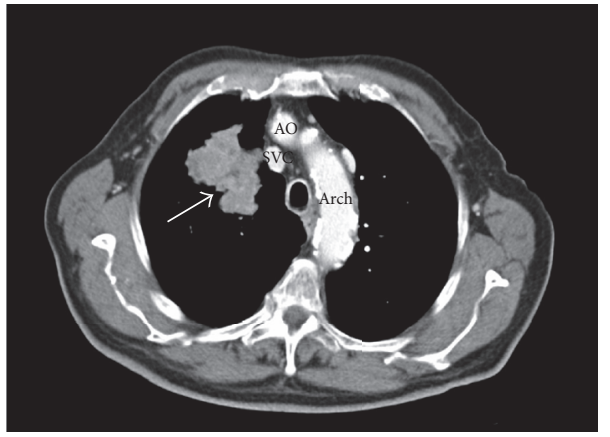

(a)

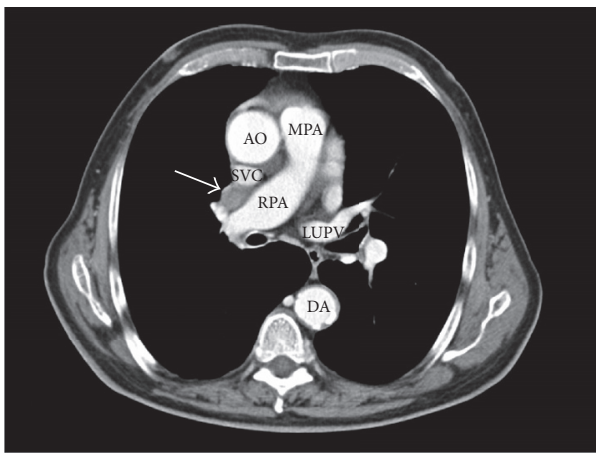

(c)

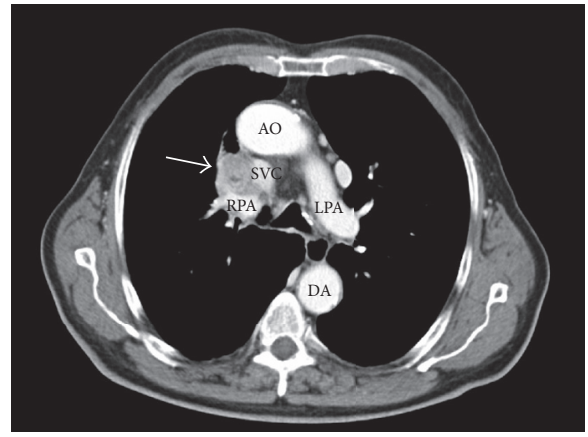

(b)

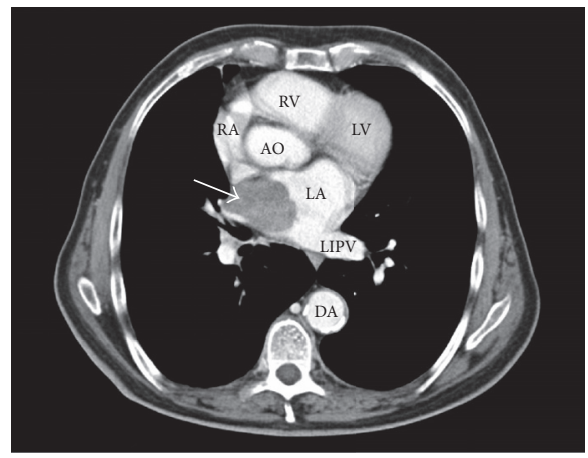

(d)

FIGURE 2: Chest CT showing a large opacity with irregular borders in the right upper lobe (a), with invasion of the right upper pulmonary vein (b-c) and extension into the left atrium (d). The arrows indicate the mass along its pathway from the right upper pulmonary lobe to the left atrium (LA). Note the lack of contrast signal in the right upper pulmonary vein, obstructed by the mass. AO, aorta; DA, descending aorta; LAA, left atrial appendage; LPA, left pulmonary artery; LIPV, left inferior pulmonary vein; LUPV, left upper pulmonary vein; LV, left ventricle; MPA, main pulmonary artery; RA, right atrium; RPA, right pulmonary artery; RV, right ventricle; SVC, superior vena cava.

the current examination, chest and cardiac examinations were normal, blood pressure was $170 / 75 \mathrm{mmHg}$, heart rate was $77 \mathrm{bpm}$, body temperature was $36.5^{\circ} \mathrm{C}$, and oxygen saturation was 95\%. The ECG was normal. Neurological examination showed facial-brachial-crural left hemiparesis (NIH Stroke Scale $=8$ ). A new brain CT showed an ipsilateral hypodense lesion in the left semioval center. The patient was hospitalized, and therapy with aspirin, methylprednisolone, ramipril, and dalteparin was started. An echo-Doppler of supra-aortic vessels showed subcritical stenosis of the right internal carotid artery and critical stenosis of the left common carotid artery with occlusion of proximal left internal carotid artery. An atherosclerotic stroke was diagnosed (ASCO A1b).

During the following hours, neurological conditions progressively improved. Routine chest X-ray evidenced a rounding mass projecting over the right upper lobe and extending towards the right upper portion of cardiac silhouette (Figure 1). Chest CT confirmed the presence of a large opacity with irregular borders, with invasion of the right upper pulmonary vein and extension into the left atrium (Figure 2). Transthoracic echocardiography showed massive LA invasion by a large, multilobed, highly mobile 


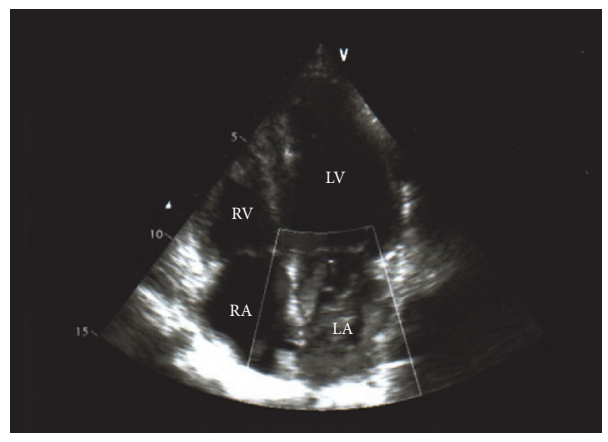

(a)

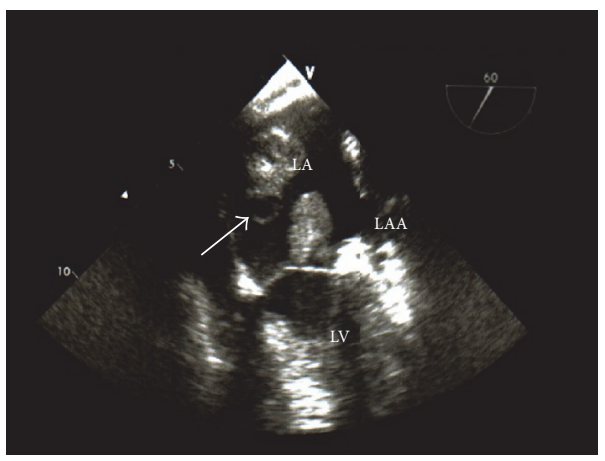

(c)

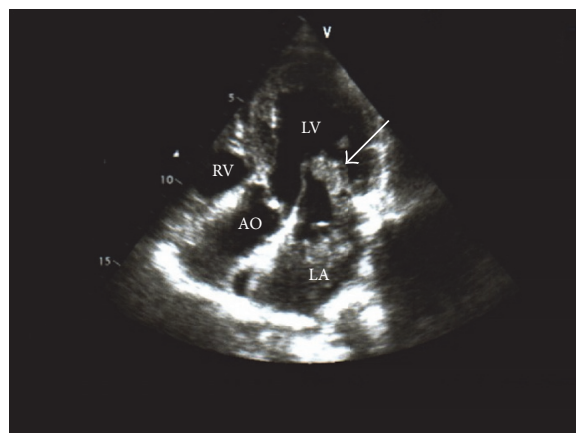

(b)

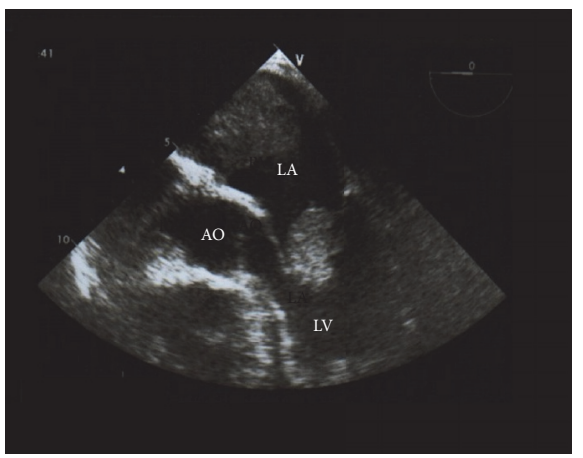

(d)

Figure 3: ((a) and (b)) Transthoracic echocardiography showing massive left atrial invasion by a large multilobed mass, with protrusion through the mitral valve into the left ventricle during diastole (arrow). ((c) and (d)) Transesophageal echocardiography confirming left atrial invasion by a large mass entering the left ventricle in diastole and showing regions of cystic colliquation within the mass (arrow). AO, aorta; LA, left atrium; LAA, left atrial appendage; LV, left ventricle; RA, right atrium; RV, right ventricle.

mass $(48 \times 35 \mathrm{~mm})$ protruding through the mitral valve into the left ventricle during diastole (Figures 3(a) and 3(b)). Transesophageal echocardiography confirmed LA invasion with occlusion of the right upper pulmonary vein and pericardial infiltration through the LA roof, allowing visualization of areas of vascularization and some regions of cystic colliquation within the mass (Figures 3(c) and 3(d)). The stroke was reclassified as $\mathrm{ASCO} \mathrm{C}_{1}$. On day 7 , sudden right hemiparesis with spatiotemporal disorientation occurred. Brain CT showed new multiple, diffuse hypodense lesions near the vertex in the left hemisphere (Figure 4). After careful clinical evaluation, the patient was considered at too high risk to undergo thoracic surgery. On day 20, cardiac magnetic resonance showed a further increase in the dimension of LA mass with subtotal obliteration of LA cavity (Figure 5). On day 23 , the patient died because of cardiac arrest.

\section{Discussion}

Although relatively rare, metastatization of pulmonary neoplasm to the left atrium has been well documented, particularly in patients with primary lung cancer $[14,15]$. In a previous review of 215 lung cancer patients studied by gadolinium-enhanced 3D magnetic resonance angiography, an involvement of the proximal portion of the pulmonary veins and an extension into the left atrium were found in

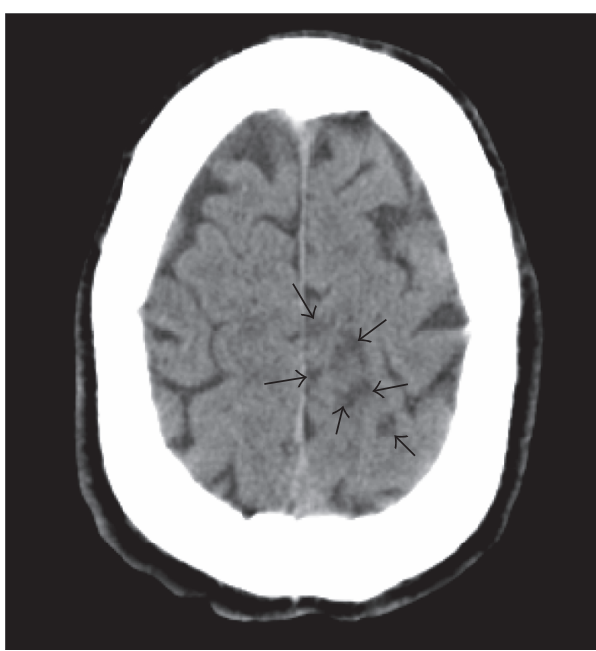

FIGURE 4: Brain CT showing new multiple, diffuse hypodense lesions near the vertex in the left hemisphere (arrows).

$9(4.2 \%)$ and $2(0.9 \%)$ patients, respectively [16]. Similarly, a more recent retrospective analysis of 4668 patients who underwent surgery for lung cancer found pathological evidence of pulmonary vein and LA involvement in $34(0.7 \%)$ and $25(0.5 \%)$ subjects, respectively [17]. LA invasion usually 


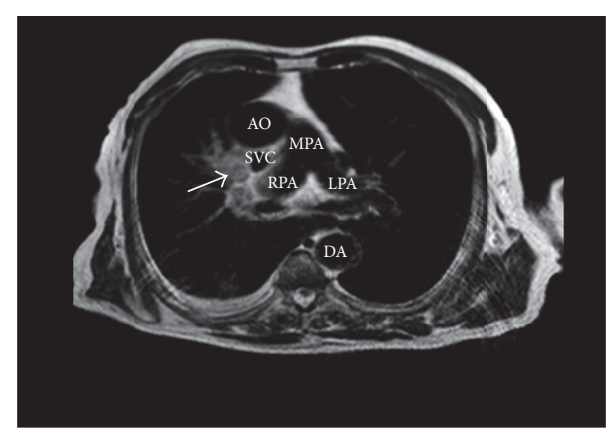

(a)

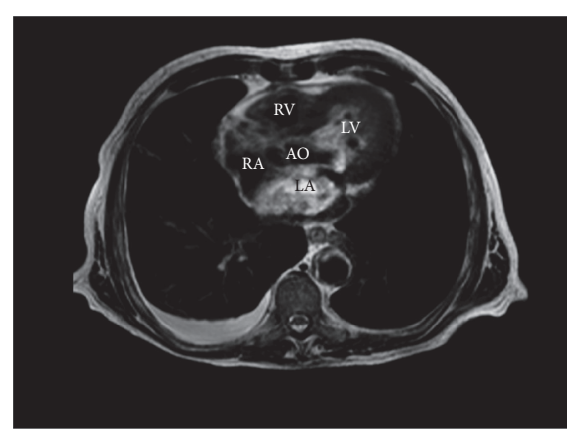

(b)

FIGURE 5: Chest magnetic resonance imaging showing invasion and subtotal obliteration of left atrial cavity from the right upper pulmonary vein (arrow). AO, aorta; DA, descending aorta; LA, left atrium; LPA, left pulmonary artery; LV, left ventricle; MPA, main pulmonary artery; RA, right atrium; RPA, right pulmonary artery; RV, right ventricle; SVC, superior vena cava.

occurs by two main mechanisms, including direct infiltration of myocardial tissue by contiguity [18-20] and extension into the left atrium via the lymphatics and/or the pulmonary veins [21-28]. Patients most commonly suffer symptoms related to lung cancer (e.g., cough, hemoptysis, and weight loss) or sometimes related to cardiac complications as the first clinical presentation. A limited number of reports previously described cardiovascular presentations secondary to systemic neoplastic embolization, including cerebral ischemia [29-31] or peripheral arterial occlusion [32]. Noteworthy, most previous reports of cerebral ischemia described events with typical contralateral presentation $[12,29]$ or incidental detection of brain ischemic lesion by imaging techniques in asymptomatic subjects [30,31]. Although patients with metastatic involvement of the heart generally have poor clinical outcome, their management should include a careful assessment of surgical options. When appropriate, the treatment of choice is complete resection in combination with chemotherapy or radiotherapy [32]. However, in the majority of cases, cardiac metastases occur in patients with advanced neoplastic disease who have already undergone resection of the tumor of origin. In these cases, cardiac treatment is usually confined to palliative interventions to relieve cardiac compression or haemodynamic obstruction if indicated. Moreover, complete resection of the tumor is not always possible, and postoperative mortality is relatively high $[33,34]$.

In this report, we describe the case of a primary lung neoplasm extending into the left atrium via a pulmonary vein and complicated by stroke presenting as ipsilateral hemiparesis. Several atypical issues should be pointed out in this case: (1) the clinical presentation as stroke with left hemiparesis and CT evidence of ipsilateral acute ischemic lesion, which might suggest the presence of uncrossed corticospinal tracts in our patient [35]; (2) the changes in stroke categorization according to the ASCO classification, related to the detection of left internal carotid artery occlusion with successive evidence of cardiac source of cerebral embolization; (3) the successive clinical evolution with sudden-onset right hemiparesis associated with multiple contralateral left-sided lesions, suggestive for an embolization pattern; (4) the growth rate of LA mass, which rapidly led to LA cavity obliteration and cardiac death. It should be pointed out that since tissue analysis data were not available, caution is needed in interpreting these findings. Although both the clinical course and imaging data support the hypothesis of a metastatic nature of the mass, without histologic confirmation, the diagnosis cannot be considered as definitively established. From a practical point of view, this report highlights the importance of considering the ASCO classification as a dynamic tool to define the phenotypic nature of stroke and of considering echocardiography as a cornerstone in the evaluation, diagnosis, and management of patients with clinical evidence of cerebral ischemia [36].

\section{Competing Interests}

The authors declare that there are no competing interests.

\section{References}

[1] B. L. Strauss, M. J. Matthews, M. H. Cohen, R. Simon, and F. Tejada, "Cardiac metastases in lung cancer," Chest, vol. 71, no. 5, pp. 607-611, 1977.

[2] K. K. Kadappu, R. Rajaratnam, H. Kachwalla, and P. D. Nguyen, "Lung cancer mimicking left atrial mass," Postgraduate Medical Journal, vol. 84, no. 993, pp. 386-387, 2008.

[3] K. Sosvińska-Mielcarek, E. Senkus-Konefka, J. Jassem, J. Kulczycka, J. Jendrzejewski, and K. Jaskiewicz, "Cardiac involvement at presentation of non-small-cell lung cancer," Journal of Clinical Oncology, vol. 26, no. 6, pp. 1010-1011, 2008.

[4] P. P. Roy, A. K. Dwari, S. K. Dey, A. Sarkar, A. Bhattacharya, and A. K. Saha, "A rare case of lung cancer with invasion in the heart giving rise to electrocardiographic features simulating myocardial infarction," Journal of the Indian Medical Association, vol. 109, no. 7, pp. 498-499, 2011.

[5] M.-S. Tsai, P. C.-I. Ko, J.-Y. Shih et al., "Cardiac involvement in malignancies. Case 1. Favorable outcome of a patient with cardiac invasion from non-small-cell lung carcinoma," Journal of Clinical Oncology, vol. 22, no. 13, pp. 2740-2742, 2004. 
[6] C.-C. Liaw, H. Chang, T.-S. Yang, and M.-S. Wen, "Pulmonary venous obstruction in cancer patients," Journal of Oncology, vol. 2015, Article ID 210916, 10 pages, 2015.

[7] R. M. Gowda, I. A. Khan, N. J. Mehta et al., "Cardiac tamponade and superior vena cava syndrome in lung cancer-a case report," Angiology, vol. 55, no. 6, pp. 691-695, 2004.

[8] K. Kinoshita, M. Hanibuchi, M. Kishi, T. Kanematsu, Y. Nishioka, and S. Sone, "Case of squamous cell lung cancer with myocardial metastasis complicated with ventricular tachycardia," The Journal of The Japanese Respiratory Society, vol. 47, no. 9, pp. 817-822, 2009.

[9] S. Morio, Y. Hara, T. Yamaga, Y. Yoshino, S. Nakamoto, and N. Sugiyoma, "A case report of complete heart block by metastatic cardiac involvement from lung cancer," The Japanese Journal of Thoracic Surgery, vol. 42, no. 11, pp. 944-947, 1989.

[10] R. R. Brandt, J. Rubin, and G. S. Reeder, "Intracardiac extension of a lung tumor causing left ventricular inflow obstruction," Journal of the American Society of Echocardiography, vol. 8, no. 6, pp. 930-933, 1995.

[11] Y. Kinjo, A. Nagasaki, I. Teruya et al., "Cardiac involvement of lung cancer presenting with acute myocardial infarction-like electrocardiographic changes," Internal Medicine, vol. 45, no. 2, pp. 113-114, 2006.

[12] A. Dimitrović, T. Breitenfeld, V. Supanc, M. Roje-Bedeković, S. Butković Soldo, and V. Vargek-Solter, "Stroke caused by lung cancer invading the left Atrium," Journal of Stroke and Cerebrovascular Diseases, vol. 25, no. 5, pp. e66-e68, 2015.

[13] P. Amarenco, J. Bogousslavsky, L. R. Caplan, G. A. Donnan, and M. G. Hennerici, "New approach to stroke subtyping: the A-S-C-O (phenotypic) classification of stroke," Cerebrovascular Diseases, vol. 27, no. 5, pp. 502-508, 2009.

[14] F. Stella, A. Dell'Amore, G. Caroli et al., "Surgical results and long-term follow-up of $\mathrm{T}_{4}$-non-small cell lung cancer invading the left atrium or the intrapericardial base of the pulmonary veins," Interactive Cardiovascular and Thoracic Surgery, vol. 14, no. 4, pp. 415-419, 2012.

[15] K. L. Chuah, W. M. Yap, H. L. Loh, K. H. Lim, H. W. Tan, and C. H. Lim, "Intravenous extension of sarcomatoid carcinoma of the lung to the left atrium," Pathology, vol. 38, no. 4, pp. 359-361, 2006.

[16] K. Takahashi, M. Furuse, H. Hanaoka et al., "Pulmonary vein and left atrial invasion by lung cancer: assessment by breathhold gadolinium-enhanced three-dimensional MR angiography," Journal of Computer Assisted Tomography, vol. 24, no. 4, pp. 557-561, 2000.

[17] M. Riquet, B. Grand, A. Arame et al., "Lung cancer invading the pericardium: quantum of lymph nodes," Annals of Thoracic Surgery, vol. 90, no. 6, pp. 1773-1777, 2010.

[18] S. Guha, S. Mookerjee, R. N. Karmakar et al., "Left atrial extension of lung malignancy with ECG changes resembling STEMI," Indian heart journal, vol. 62, no. 1, pp. 81-83, 2010.

[19] J. Shimizu, C. Ikeda, Y. Arano et al., "Advanced lung cancer invading the left atrium, treated with pneumonectomy combined with left atrium resection under cardiopulmonary bypass," Annals of Thoracic and Cardiovascular Surgery, vol. 16, no. 4, pp. 286-290, 2010.

[20] A. Ucak, K. Inan, B. Onan, V. Temizkan, I. Alp, and A. T. Yilmaz, "Free-floating tumor thrombus in the left atrium associated with non-small cell lung cancer," Journal of Cardiac Surgery, vol. 24, no. 6, pp. 686-689, 2009.

[21] V. Chan and D. Neumann, "Small cell lung carcinoma invading the pulmonary vein and left atrium as imaged by PET/CT,"
European Journal of Nuclear Medicine and Molecular Imaging, vol. 32, no. 12, p. 1493, 2005.

[22] Y. Funakoshi, T. Mukohara, T. Kataoka et al., "Left atrial extension of metastatic lung tumor via pulmonary vein: report on the first case of Ewing sarcoma," Rare Tumors, vol. 2, no. 3, article no. e53, 2010.

[23] J. H. Woodring, B. Bognar, and C. S. Van Wyk, "Metastatic chondrosarcoma to the lung with extension into the left atrium via invasion of the pulmonary veins: presentation as embolic cerebral infarction," Clinical Imaging, vol. 26, no. 5, pp. 338-341, 2002.

[24] M. A. Jadoon and P. Sidhu, "Advanced right lung adenocarcinoma invading left atrium and left ventricle via right superior pulmonary vein and partially occluding mitral valve in diastole," European Journal of Echocardiography, vol. 12, no. 6, article 420, 2011.

[25] M. Y. Desai and S. Mankad, "Extension of bronchogenic carcinoma through pulmonary vein into the left atrium detected by echocardiography," Echocardiography, vol. 21, no. 2, pp. 189-191, 2004.

[26] T. W. Koh, "Invasion of lung mesenchymal chondrosarcoma into the left atrium via the pulmonary vein detected on transoesophageal echocardiography," European Journal of Echocardiography, vol. 12, no. 7, p. 556, 2011.

[27] A. G. Pitman, B. Solomon, R. Padmanabhan, A. F. Mckenzie, and R. J. Hicks, "Intravenous extension of lung carcinoma to the left atrium: demonstration by positron emission tomography with CT correlation," British Journal of Radiology, vol. 73, no. 866, pp. 206-208, 2000.

[28] N. Watanabe and K. Kubo, "Images in cardiology: intra-left atrial invasive mass extended via the pulmonary vein," Heart, vol. 85 , no. 3, p. 271, 2001.

[29] L. Ascione, G. Granata, M. Accadia, G. Marasco, R. Santangelo, and B. Tuccillo, "Ultrasonography in embolic stroke: the complementary role of transthoracic and transesophageal echocardiography in a case of systemic embolism by tumor invasion of the pulmonary veins in a patient with unknown malignancy involving the lung," European Journal of Echocardiography, vol. 5, no. 4, pp. 304-307, 2004.

[30] G. F. Gates, A. Aronsky, and H. Ozgur, "Intracardiac extension of lung cancer demonstrated on PET scanning," Clinical Nuclear Medicine, vol. 31, no. 2, pp. 68-70, 2006.

[31] H. Oizumi, R. Tanaka, H. Shimura et al., "A case of cerebral embolism with metastatic chondrosarcoma in the left atrium," Journal of Stroke and Cerebrovascular Diseases, vol. 20, no. 1, pp. 79-81, 2011.

[32] K. Reynen, U. Köckeritz, and R. H. Strasser, "Metastases to the heart," Annals of Oncology, vol. 15, no. 3, pp. 375-381, 2004.

[33] U. Andrushchuk, Y. Ostrovsky, V. Zharkov et al., "Surgery for massive malignant tumors of the left atrium-one center's experience," Polish Journal of Cardio-Thoracic Surgery, vol. 13, no. 3, pp. 229-235, 2016.

[34] T. Strecker, J. Rösch, M. Weyand, and A. Agaimy, "Primary and metastatic cardiac tumors: Imaging characteristics, surgical treatment, and histopathological spectrum: a 10-yearexperience at a German heart center," Cardiovascular Pathology, vol. 21, no. 5, pp. 436-443, 2012.

[35] A. S. L. Ng, Y.-Y. Sitoh, Y. Zhao, E. W. L. Teng, E. K. Tan, and L. C. S. Tan, "Ipsilateral stroke in a patient with horizontal gaze palsy with progressive scoliosis and a subcortical infarct," Stroke, vol. 42, no. 1, pp. el-e3, 2011. 
[36] M. Pepi, A. Evangelista, P. Nihoyannopoulos et al., "Recommendations for echocardiography use in the diagnosis and management of cardiac sources of embolism: European Association of Echocardiography (EAE) (a registered branch of the ESC)," European Journal of Echocardiography, vol. 11, no. 6, pp. 461476, 2010. 


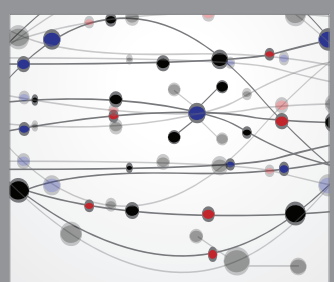

The Scientific World Journal
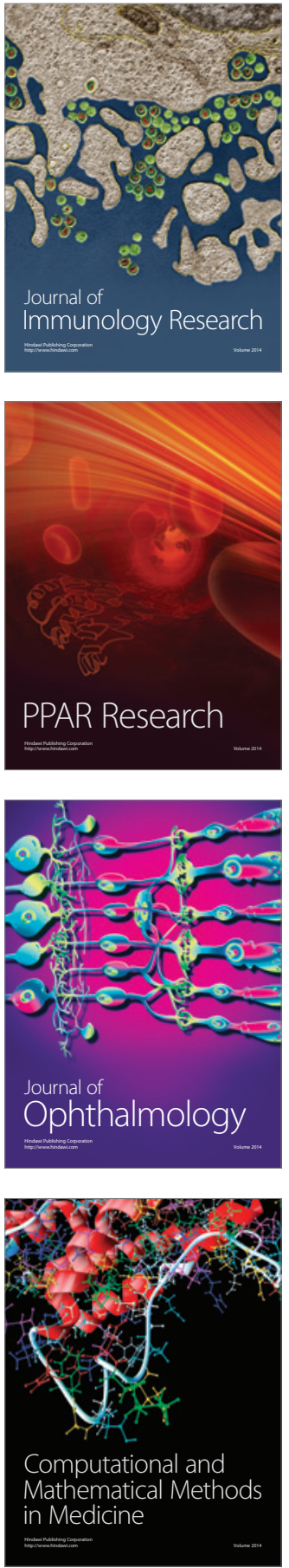

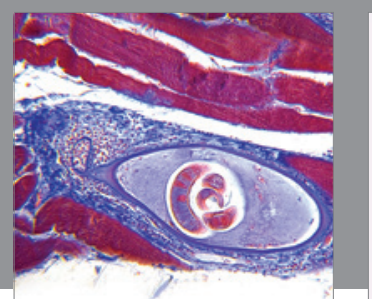

Gastroenterology Research and Practice

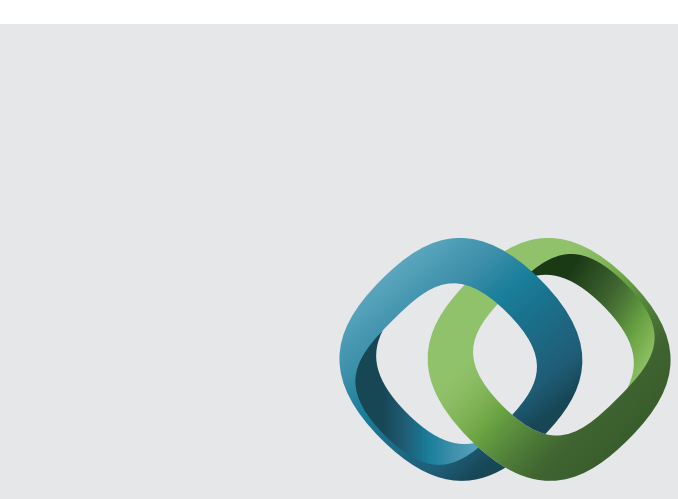

\section{Hindawi}

Submit your manuscripts at

http://www.hindawi.com
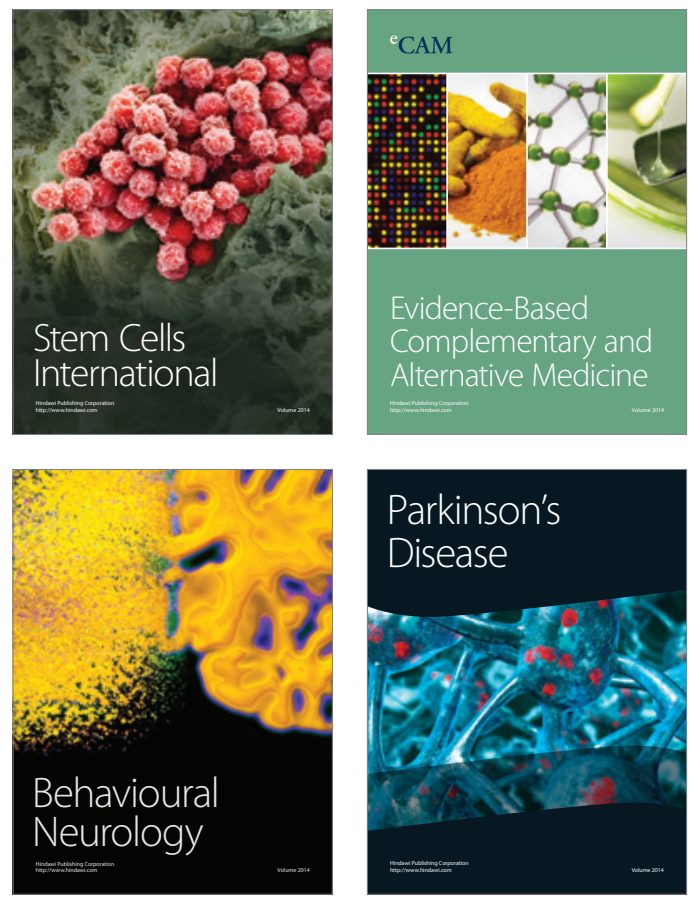
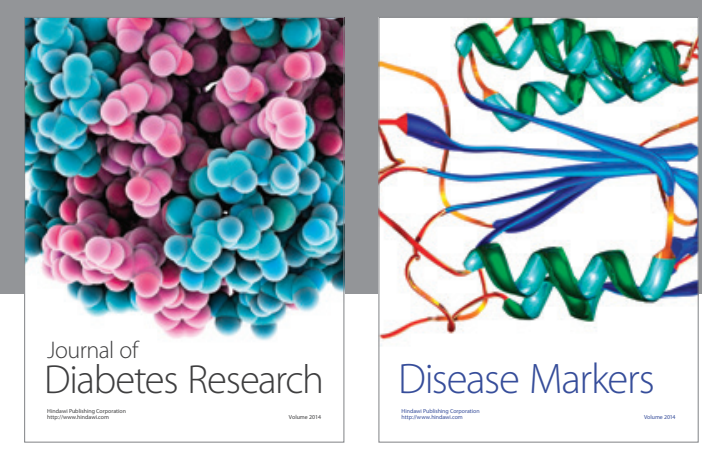

Disease Markers
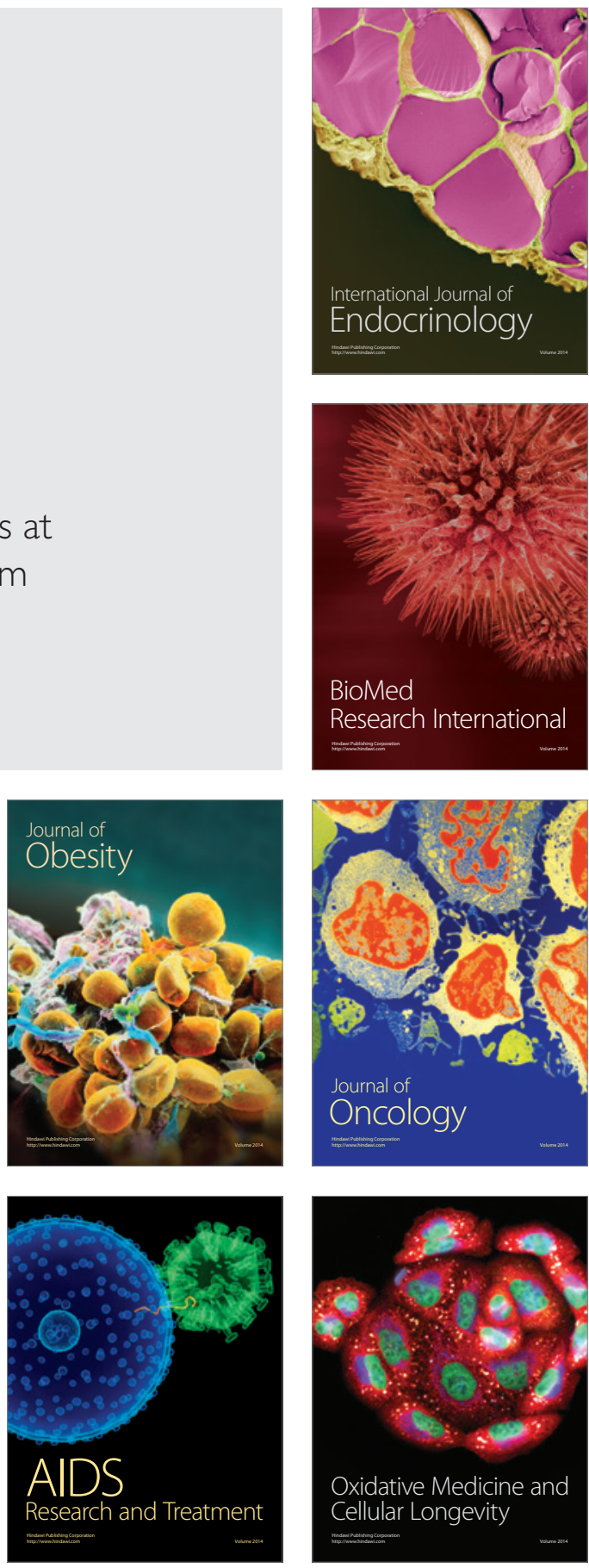